\title{
Evolución general de la economía salvadoreña durante 1989
}

\author{
Ulises Valenzuela
}

\section{Introducclón}

En términos generales, la economía salvadorefia habría continuado durante 1989 con su tendencia de crisis y estancamiento que ha presentado durante los últimos 10 años. La producción nacional apenas habría crecido en términos globales, mientras que en términos per cápita habria disminuido por décimo primer año consecutivo y retrocedido a los niveles de hace más de veinticinco ahos.

Enfocada desde una perspectiva de estabilidad, aunque el proceso inflacionario habria sido aparentemente menor que el de los últimos cuatro años, la situación económica en general se habria agudizado. En realidad, el proceso inflacionario incluso se habría acelerado, si lo medimos por la tasa acumulada de inflación. Como quiera que sea, lo cierto es que las condiciones de vida de la mayoría de la población habrían continuado su acelerado deterioro. Los desequilibrios fiscales y externo, por su parte, no sólo se habrían agudizado, sino alcanzado en algunos casos niveles nunca antes observados.

Lo que a continuación se presenta es principalmente una descripción general de la evoución de la economía durante 1989 y de algunas de sus principales tendencias. Aunque a la par se presentan también algunas valoraciones sobre la marcha hasta ahora del programa económico del actual gobierno, cabe advertir que ésto no constituye en modo alguno una evaluación, ni siquiera parcial o preliminar, del mismo.

\section{Producclón y empleo}

Siguiendo una tendencia hacia el estancamiento que ha presentado 
durante casi toda la década, según cifras preliminares del BCR, durante 1989 el PIB habria crecido en apenas un $1 \%-0.6$ puntos porcentuales menos que el año anterior-, de manera que el PIB per cápita habría caído por décimo primer año consecutivo, esta vez en un $1.8 \%$ aproximadamente. Por el lado de la demanda, lo que explicaría el reducido dinamismo de la actividad económica en 1989 seria, en primer lugar, una drástica caida en las exportaciones de bienes y servicios (cuadro 1), vinculada estrechamente a la súbita baja experimentada por los precios del café, que provocó en parte la ruptura del acuerdo sobre / cuota de OIC en el mes de julio. La caída de las exportaciones del grano se debió también a una reducción de casi un $43 \%$ en la producción de la cosecha 1988-89.

El componente más dinámico de la demanda habria sido la formación de capital fijo, sobre todo privado (cuadro 1). Sin embargo, y a diferencia del consumo, el dinamismo que este componente mostrara desde 1984 ha venido disminuyendo. La práctica en los últimos años de una política monetario-crediticia bastante restrictiva (cuadro 2) sería responsable en parte del desempeño de la inversión privada. En 1989, y de manera especial en su segunda mitad, las autoridades monetarias habrian seguido una política crediticia severamente restrictiva hacia el sector privado, que habria contribuido nuevamente al reducido dinamismo de ese importante rubro. El ambiente político prevaleciente previo a las elecciones de marzo, el recrudecimiento de la guerra durante el último bimestre del año y la continuación en general del ambiente político-militar que ha imperado en el pais durante los últimos diez años, incidieron también de manera sustancial sobre el comportamiento de la inversión privada. Estos factores prevalecieron sin duda sobre el entusiasmo despertado en el gran capital por el triunfo presidencial de su proyecto político-ideológico.

Por el lado de la oferta, el grave estancamiento del sector agropecuario, y en especial el retroceso de la agricultura, contribuyeron en parte al escaso dinamismo del PIB. Es importante destacar la crisis en que el sector agropecuario en general (cuadro 1) y la agricultura en particular, se encuentran sumidos. En los últimos cinco años, la agricultura ha experimentado invariablemente tasas negativas de crecimiento. La producción real de este sub-sector en 1989 representó menos de las tres cuartas partes de la de 1980, como puede apreciarse en el gráfico 1.

La reactivación de la agricultura parece plantear un serio reto para el actual gobierno, que, aparte de la liberalización de precios y algunas líneas especiales de crédito, no parece contar con una estrategia 
Cuadro 1

Oferta y demanda global real: tasas de crecimiento

\begin{tabular}{lrrrr}
\hline & 1986 & 1987 & 1988 & 19891 \\
\hline DEMANDA GLOBAL & 0.0 & 2.3 & 1.2 & 1.1 \\
CONSUMO & 0.5 & 1.1 & 1.4 & 1.7 \\
Privado & -0.3 & 0.6 & 1.1 & 1.5 \\
Público & 3.7 & 3.0 & 2.5 & 2.6 \\
INVERSION INTERNA BRUTA & 21.5 & -4.2 & 18.5 & 15.7 \\
Formación de Capital Fijo & 7.5 & 9.1 & 2.4 & 3.0 \\
Privado & 10.1 & 7.3 & -0.6 & 4.1 \\
Público & -15.8 & 14.4 & 10.7 & 0.2 \\
Variación en Inventarios & & & & \\
EXPORTACIONES & -12.6 & 12.4 & -9.4 & -12.6 \\
OFERTA GLOBAL & 0.0 & 2.3 & 1.2 & 1.1 \\
IMPORTACIONES & -2.8 & 0.4 & -0.5 & 1.8 \\
PRODUCTO INTERNO BRUTO & 0.6 & 2.7 & 1.6 & 1.0 \\
Agropecuario & -3.1 & 2.1 & -1.0 & 0.2 \\
Mineria y Canteras & 2.6 & 4.7 & 4.7 & 0.0 \\
Industria Manufacturera & 2.5 & 3.0 & 3.0 & 0.8 \\
Construcción & 2.6 & 11.5 & 5.6 & 3.8 \\
Electricidad y agua & 2.5 & 2.0 & 1.8 & 0.8 \\
Transporte, almacenaje y Com. & 0.5 & 1.8 & 2.0 & 1.2 \\
Comercio & 0.3 & 1.4 & 0.4 & 0.3 \\
Financiero & 1.5 & 2.4 & 2.0 & 0.6 \\
Propiedad de viviendas & 0.3 & 2.6 & 4.4 & 1.6 \\
Administración pública & 0.5 & 4.0 & 3.4 & 2.8 \\
Servicios personales & 0.5 & 1.5 & 1.4 & 0.2 \\
\hline
\end{tabular}

Fuente: Banco Central de Reserva

1. Estimación (incluyen daños de ofensiva FMLN).

\section{Cuadro 2}

Crédito y medlos de pago: tasas de crecimiento en términos reales

\begin{tabular}{lrrrr}
\hline & 1986 & 1987 & 1988 & 1989 \\
\hline Crédito Total & -26.1 & -5.6 & -4.6 & 2.3 \\
$\quad$ Sector Privado & -17.8 & -5.7 & -4.6 & -14.3 \\
Sector Público & -44.5 & -5.4 & -9.7 & 39.7 \\
& & & & \\
Medios de Pago & -7.9 & -6.4 & -4.7 & -5.8 \\
$\quad$ Medios Circulantes & -15.9 & -13.4 & -8.5 & -3.7 \\
$\quad$ Cuasi-Dinero & -2.6 & -1.9 & -2.5 & -7.0 \\
\hline
\end{tabular}

Fuente: Calculado con base en cifras del BCR. 
definida para revivir al sub-sector como un todo. Las autoridades económicas, más interesadas en promover exportaciones que en la producción de bienes agricolas básicos, no parecen haberse percatado de las dimensiones de este problema, y raras veces se refieren a la crisis de la agricultura en general; si son frecuentes sus alusiones a los problemas de la caficultura. Parece haber, en ese sentido, muy poco

\section{Gráflco 1 \\ EVOLUCION DE LA AGRICULTURA \\ $(1980=100)$}

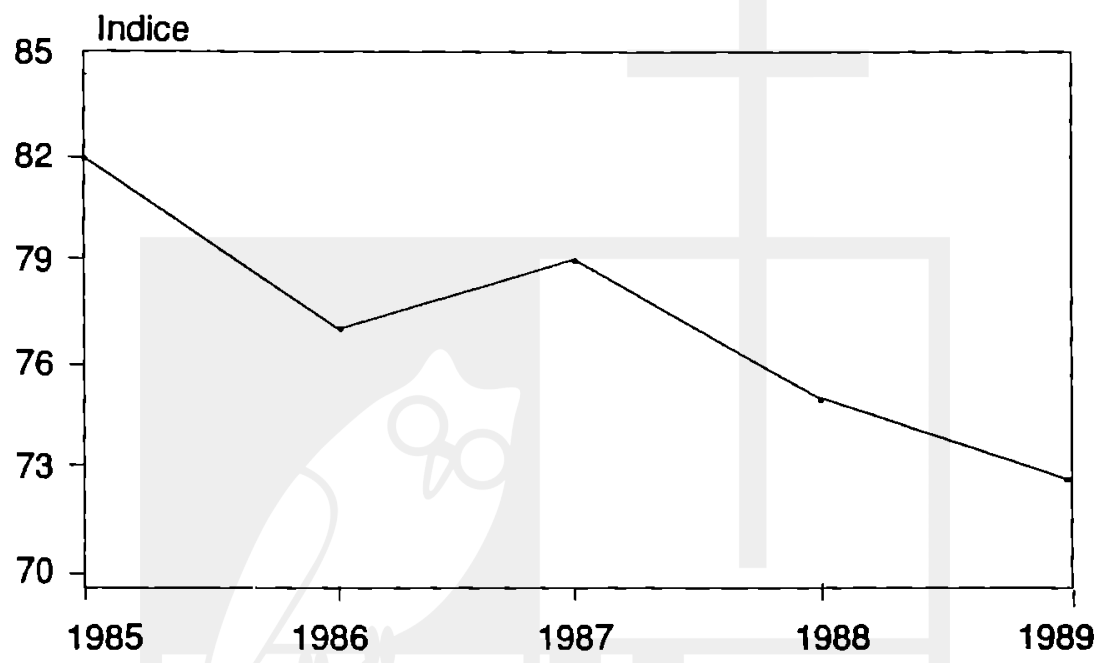

interés en la promoción de producción de bienes agrícolas no exportables, por más que estos sean indispensables para el consumo básico de las mayorías. Aunque el gobierno tiene en principio un reducido margen de maniobra en el manejo de este problema mientras la variable clave en la crisis agricola - la guerra- siga presente, su poco interés no contribuye sino a agravar el problema.

Las implicaciones del problema no se reducen únicamente a su incidencia sobre el crecimiento de la economia en general, sino también sobre su estabilidad. En efecto, se ha logrado establecer que este es un problema que tiene repercusiones nada desdeñables sobre la inflación y la balanza de pagos'. El impacto sobre esta última probablemente no se manifieste con suficiente claridad por ahora, debido en parte a las fuertes donaciones que el pais ha estado recibiendo del exterior en 
ayuda alimentaria. En cuanto a la inflación, en cambio, el retroceso en la producción agricola básica pudiera estar jugando ya un papel importante en el repunte inflacionario observado en los últimos anos en el rubro de alimentos (véase gráfico 2, más adelante).

El sector industria manufacturera, por su parte, que en los últimos 5 af́os habia venido creciendo bastante por encima del PIB (cuadro 1), experimentó una desaceleración bastante significativa. Esto puede deberse tanto a un menor crecimiento de la inversión en el sector, como a las mayores dificultades para importación de insumos generada por el alza en el tipo de cambio, y a la política restrictiva practicada por el gobierno hacia el sector privado. La menor protección para el sector que la liberalización de la importación de algunos bienes de origen industrial ha implicado, pudiera también estar empezándose a sentir.

El sector construcción también ha mostrado en 1989 un dinamismo menor, si bien ha continuado siendo el sector con una mayor tasa de crecimiento (cuadro 1). El carácter restrictivo de la política crediticia hacia el sector privado, así como mayores tasas de interés, un tipo de cambio superior y el recrudecimiento de la guerra incidieron indudablemente en este resultado. A medida que el tipo de cambio siga aumentando y el crédito se vuelva relativamente más caro -como habria de suceder a medida que el gobierno avance en la implementación de su programa de ajuste estructural-, este sector podría convertirse en uno de los grandes perdedores de la nueva década. Dentro del esquema de promoción de explortaciones y de los intentos por reducir el sesgo antiexportador de la economía salvadoreńa, el sector construcción no tiene evidentemente ninguna prioridad, por más grande que sean las necesidades que en el pais existan al respecto.

En lo que concieme al desempleo, aunque al parecer no existen aun datos para 1989, el estancamiento de la producción sugiere que es muy poco probable que haya disminuido, y que inclusive puede muy bien haber aumentado. $Y$ este es sin duda uno de los más graves problemas que el gobierno ha de afrontar. De acuerdo con datos del MIPLAN, en 1988 el desempleo y subempleo urbano alcanzaba el $51 \%$ de la PEA, mientras que en el área rural la cifra era de $71 \%$. Las cifras generales eran de un desempleo abierto de $9.4 \%$ y una tasa de subempleo del $50 \%{ }^{2}$. Las cifras correspondientes a 1987 eran de 10.9 y $47.9 \%$, respectivamente. Así, de ser estos datos correctos, a un crecimiento de $1.6 \%$ en 1988, habria correspondido una reducción de 1.5 puntos porcentuales en el nivel de desempleo abierto y un aumento de 2.1 en el subempleo. No parecen haber mayores razones para pensar que esta relación no se haya mantenido en lo fundamental en 1989. 
Vale la pena sefialar que en este punto el gobierno se entrenta con algunos incómodos dilemas. Por un lado, le es de primera prioridad el reducir el subempleo y desempleo. Pero, por otro lado, es casi igualmente prioritario para él reducir el déficit fiscal y la inflación; esto último aún a costas de cierto sacrificio en términos de crecimiento económico, como lo demuestra la conservadora política monetariacrediticia que se ha seguido no sólo durante 1989, sino también en los últimos cuatro años (véase cuadro 2, y gráfico 3 más adelante). Esto lo deja en realidad con muy poco margen de maniobra -al menos en el corto plazo-, mientras la economia no sea estabilizada: Por una parte, no puede emprender por su propia cuenta programas amplios $-\mathrm{y}$ no digamos masivos- de empleo, dada su política fiscal -que veremos con mayor detalle más adelante - de restringir el gasto y de reducir la carga tributaria de la economia. El incremento del déficit fiscal que un programa asi generararia, en un contexto de disminución del financiamiento extemo, presionaría el nivel general de precios hacia el alza.

Esto significa que el problema queda fundamentalmente en manos del sector privado, para el cual el aumento del empleo en si no puede ser el criterio prioritario de inversión. $Y$ si las autoridades monetarias continúan con la política restrictiva seguida en 1989 por temor a acelerar la inflación y a generar presiones alcistas en el tipo de cambio, el horizonte de posibilidades para disminuir el desempleo-subempleo se torna bastante estrecho. Esto sin tomar en cuenta la "inercia" hacia el estancamiento económico que la sola continuación del conflicto bélico genera.

\section{Preclos y salarlos}

En cuanto a precios, las cosas parecen haber marchado un tanto mejor. La inflación durante 1989 habria sido de 17.6\%, una tasa ligeramente inferior a la programada por las autoridades económicas $(18 \%)$. Esta constituiria la tasa más baja de inflación experimentada por la economia salvadoreña desde 1985. Asumiendo que esta cifra es la correcta (o verdadera), la reducción - aunque leve- del proceso inflacionario seria un logro de relativa importancia para 1989, sobre todo si se tiene en cuenta la devaluación sufrida por el colón durante el año - que pudiera andar en por lo menos un $25 \%^{3}$-, la liberalización de los precios de más de 250 bienes en la segunda mitad del año y la magnitud del crédito otorgado por el BCR al gobierno central (véase apartado 4).

Este logro, sin embargo, pareciera no tener mucho que ver con la política económica y de liberalización que comenzó a implementar el 
actual gobierno tras su llegada al poder. En efecto, la inflación experimentada entre junio y diciembre es 2.5 veces superior a la observada entre diciembre 1988 y mayo 1989 (cuadro 3). La tasa anualizada de inflación correspondiente a los últimos 7 meses de 1989 fue de $32.4 \%$, sin duda una de las más altas durante toda la década para un período similar. El argumento de que todos los males actuales y por llegar se deben a la "gestión irresponsable y corrupta" del gobiemo anterior, esgrimido permanentemente por las actuales autoridades económicas,

\section{Cuadro 3}

Tasa de Inflación (")

\begin{tabular}{lrr}
\hline & 1988 & 1989 \\
\hline TASA MEDIA ANUAL & 19.8 & 17.6 \\
TASA ACUMULADA & & \\
Diciembre-diciembre & 18.2 & 23.5 \\
Diciembre-mayo & 8.9 & 6.2 \\
Mayo-diciembre & 6.5 & 16.2 \\
\hline
\end{tabular}

Fuente: DIGESTYC

(*) Medida por la tasa de variación del IPC

carece acá de toda validez.

Cabe notar que pese, a la situación creada por la ofensiva guerrillera de noviembre-diciembre, no parece que los precios hayan subido notablemente durante ese bimestre, ni que hayan diferido sustancialmente en su comportamiento con respecto a afios anteriores. Aunque la oportuna intervención del Ministerio de Economía tendría algo que ver con ello, el fenómeno parece estar más asociado a los fuertes flujos de ayuda externa en especie que en ese periodo recibió el pais.

No todos los precios, sin embargo, han marchado dentro de los márgenes aceptables. Particularmente seria es la evolución seguida por el rubro alimentos. Como lo muestra el gráfico 2, la brecha entre el indice general y el de alimentos, que viene ampliándose desde 1987, se ensanchó de manera bastante significativa en 1989, especialmente durante su segunda mitad. Este problema está vinculado no sólo a la liberalización de precios para muchos bienes alimenticios y al alza del tipo de cambio (sobre todo a partir de julio), sino también a la crisis en la que -como ya hemos visto- se encuentra sumida la agricultura y al escaso dinamismo (o estancamiento relativo) de la producción industrial de alimentos.

Es interesante observar la evolución seguida por los precios en los 


\section{Gráfico 2}

EVOLUCION DEL IPC 1989 (1978 = 100)

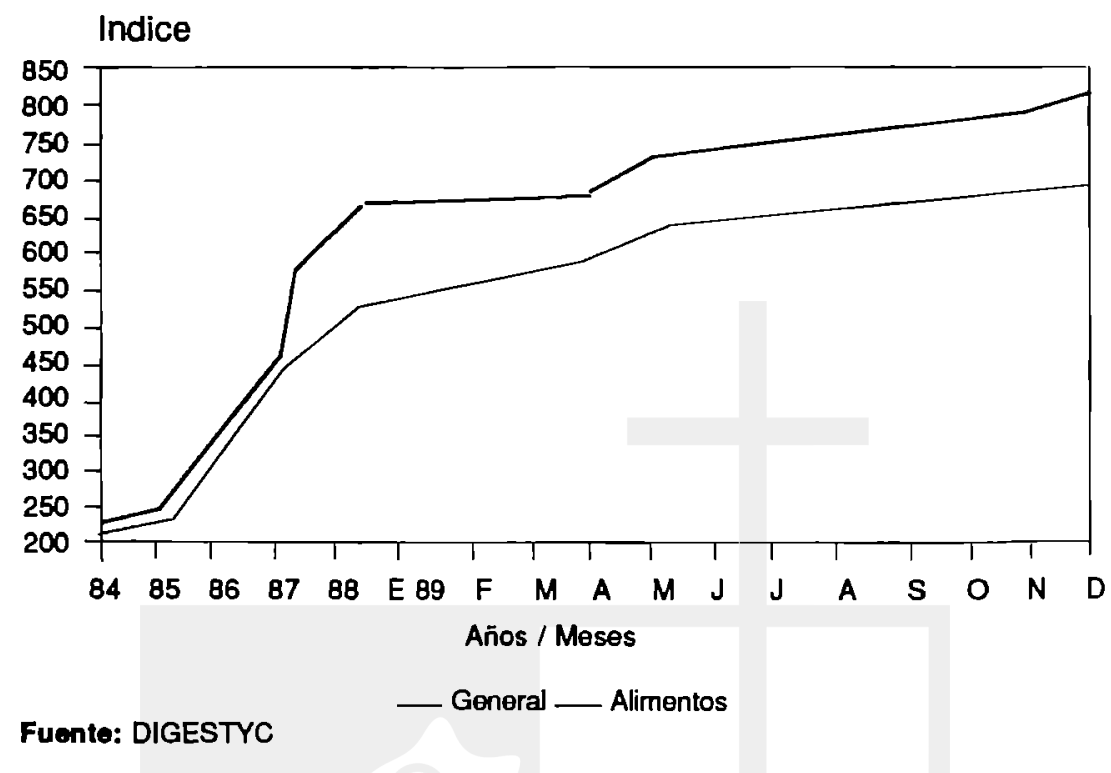

\section{Gráfico 3}

\section{RELACION OFERTA MONETARIA/PIB (\%)}

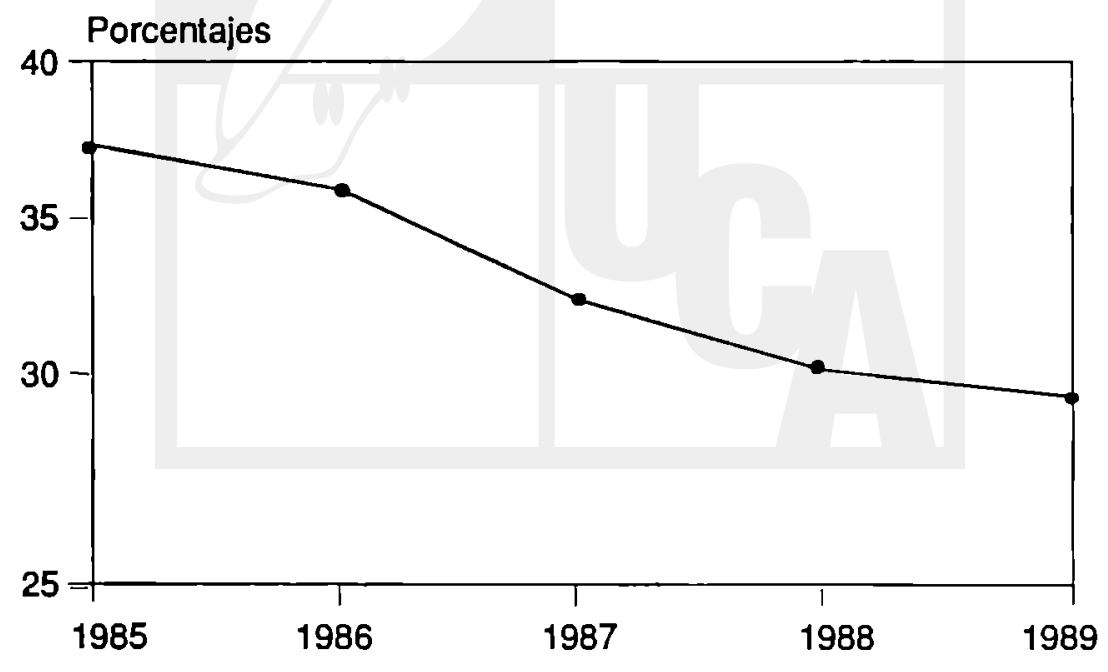

Fuente: BCR. 
últimos años frente a la de la política monetaria. Asi, mientras entre 1986 y 1989, la oferta monetaria real se ha contraído invariablemente y la relación oferta monetaria/PIB ha disminuido en más de 10 puntos durante ese periodo (veáse gráfico 3), la inflación no ha podido ser controlada efectivamente. Como se ha establecido en algunos estudios ${ }^{4}$, la inflación en El Salvador no parece ser resultado de una política macroeconómica irresponsable, sino un problema de costos, expectativas inflacionarias y ciertas rigideces estructurales.

Antes de referirnos a la evolución seguida por los salarios reales, conviene formular algunas observaciones sobre lo que en realidad mide la tasa media de inflación anual, que es la que generalmente se usa para medir el movimiento de los precios. Como se sabe, esta tasa se calcula a partir de la inflación de cada mes con respecto al mismo mes del año anterior, y el promedio de la variación por mes constituye la tasa media anual. Esta tasa, por lo tanto, no refleja la tendencia seguida por los precios durante el año en cuestión, sino su tendencia con relación al año anterior. Consiguientemente, no es el mejor indicador para medir la evolución de los salarios o ingresos reales durante un año

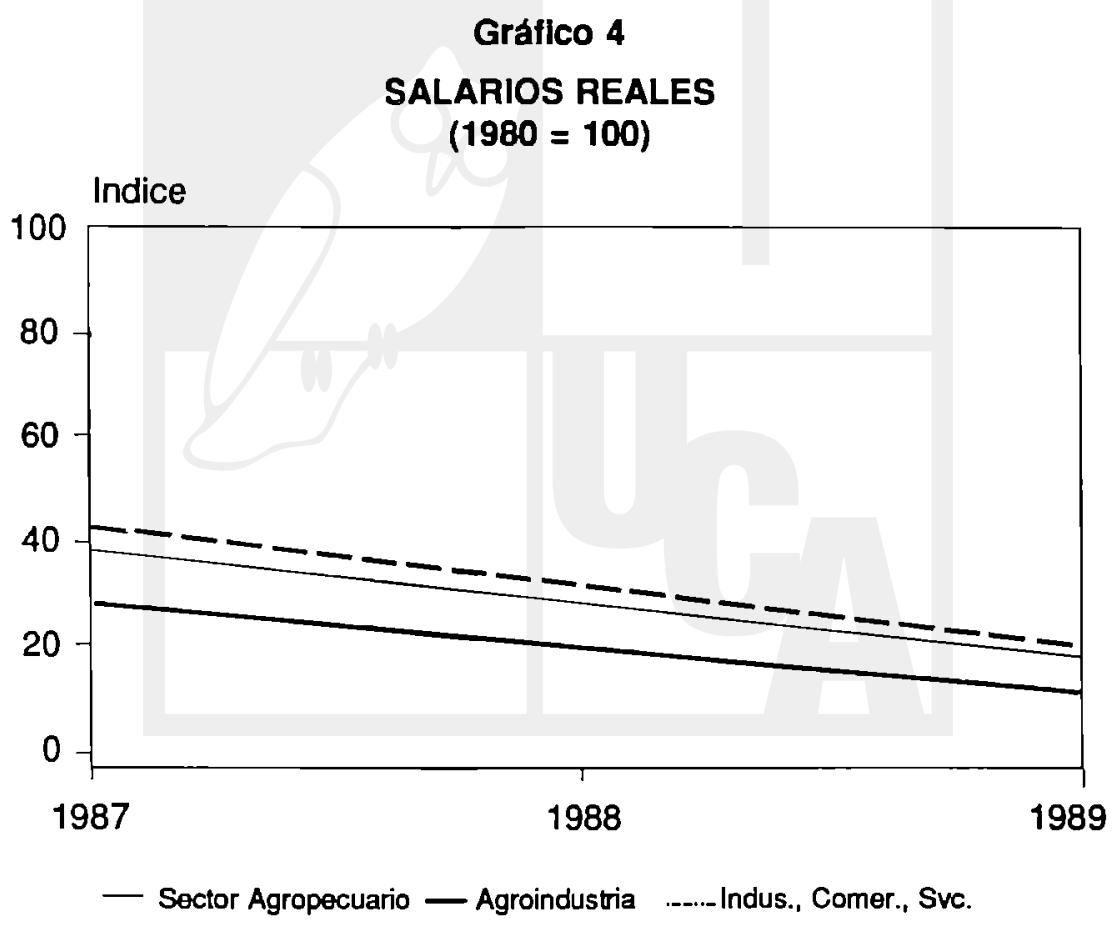

Fuente: DIGESTYC y Ministerio de Trabajo. 
determinado (o su situación a final de año).

La tasa acumulada (de diciembre a diciembre) de inflación, en cambio, mide la evolución de los precios durante el año, y es un mejor indicador sobre la verdadera evolución de los salarios o ingresos reales. Para 1989, la tasa acumulada fue superior a la media en casi 6 puntos porcentuales y mostró una tendencia ascendente con relación al año anterior (cuadro 3); además de ser la mayor desde 1986, año en que tuvo lugar la inflación más alta de la década.

En el gráfico 3 puede observarse el grave deterioro que el salario minimo real —calculado a partir de la tasa acumulada de inflación- ha sufrido durante los últimos 3 años. Como puede verse, es durante 1989 que este salario sufre su más agudo deterioro. Nótese que el salario mínimo real para este año es inferior en casi un $70 \%$ en la agroindustria, la industria, el comercio y los servicios, y en casi un $80 \%$ en el sector agropecuario, al que prevalecia hace una década. En cuánto a la evolución de los salarios reales en general, teniendo en cuenta el incremento de 200 colones a los empleados públicos que tuvo lugar en junio, y que al parecer no ha tenido lugar una medida similar en el resto de la economía, el poder adquisitivo de los salarios nominales en general se habria deteriorado en no menos de un $20 \%$ durante el afio.

En realidad, en el programa de ajuste estructural que ha comenzado a ser implementado, no parece haber una política seria tendiente a la protección del salario real. Mas aún, de acuerdo con los teóricos de los programas de ajuste estructural, la caída de los salarios reales es de hecho un elemento necesario para mejorar las posibilidades de éxito, en cuanto a estabilización y crecimiento, de los mismos ${ }^{5}$. Desde esta perspectiva, y por más injusto que pueda ser el hecho de que los más pobres tengan que cargar con los costos del ajuste, las autoridades económicas ven como un mal necesario, o inclusive con buenos ojos, la caida de los salarios reales, aunque obviamente no sus implicaciones políticas.

\section{Sector externo}

\subsection{Balanza de pagos}

A pesar de la devaluación sufrida por el colón durante el año y la práctica de una política crediticia restrictiva, el sector externo de la economia salvadorehá sutrió un fuerte deterioro en 1989. Según cifras preliminares del BCR, el déficit en la balanza comercial alcanzó un nivel sin precedentes de 603 millones de dólares, mientras la cuenta corriente, 


\section{Cuadro 4}

\section{Balanza de pagos}

(millones de dólares)

\begin{tabular}{lrrrr}
\hline & 1986 & 1987 & $19888^{1}$ & 19891 \\
\hline Exportaciones de Bienes & 754.9 & 590.9 & 608.8 & 498.8 \\
Importaciones de Bienes & 934.9 & 994.1 & 1006.9 & 1106.1 \\
Balanza comercial & -180.0 & -403.2 & -398.1 & -607.3 \\
Servicios Netos & -87.4 & -30.4 & -58.1 & -39.2 \\
Transferencias Netas & 304.0 & 573.2 & 508.6 & 519.2 \\
Cuentas Corrientes & 116.6 & 139.6 & 52.4 & -127.3 \\
Cuentas de Capital & 30.2 & -72.6 & -145.1 & 91.2 \\
$\quad$ Capital Oficial & 64.0 & 57.6 & 62.0 & 99.0 \\
$\quad$ Capital Bancario & -74.0 & -96.2 & -46.4 & -39.3 \\
$\quad$ Capital Privado & -50.5 & -34.0 & -160.7 & -82.3 \\
Saldo Balanza de Pagos & 146.8 & 67.0 & -92.7 & -36.1 \\
$\quad$ Pagos Diferidos & -71.4 & -16.1 & 28.8 & 103.0 \\
$\quad$ Variación RIN & -75.4 & -50.9 & 63.9 & -66.9 \\
Saldo Pagos Diferidos & 22.3 & 6.2 & 35.0 & 138.0 \\
\hline
\end{tabular}

Fuente: Banco Central de Reserva.

1. Citras Preliminares

2. Para 1989 incluye revalorización del oro y del capital bancario mismo.

3. Incluye Errores y Omisiones.

por primera vez desde 1985, arrojó un saldo negativo de 127 millones de dólares, pese a los fuertes flujos de transferencias que el pais continuó recibiendo. Un mayor ingreso neto de capitales y una fuerte acumulación de pagos diferidos habrían hecho disminuir el déficit en la balanza de pagos con respecto a 1988 en casi 57 millones de dólares. Sin embargo, el país habria perdido casi 67 millones de dólares en reservas internacionales netas. La brecha exłerna, por otra parte, habría dado un salto considerable, como puede apreciarse en el grático 5.

Tanto la caída de los precios intemacionales del café, como la liberalización parcial de las improtaciones tuvo bastante que ver con el agudo deterioro de la balanza comercial y la cuenta corriente. Resultado de la ruptura del acuerdo de la Organización Internacional de Café, los precios de ese grano alcanzaron en 1989 su más bajo nivel en 15 años, al caer de aproximadamente 165 dólares el quintal, en que se encontraban en el mes de enero, a menos de 70 dólares en octubre pasado. Esta habría sido la causa principal de la caída de las exportaciones del grano en casi un $36 \%$ en dólares, alcanzando su más 


\section{Gráfico 5}

\section{BRECHA EXTERNA*}

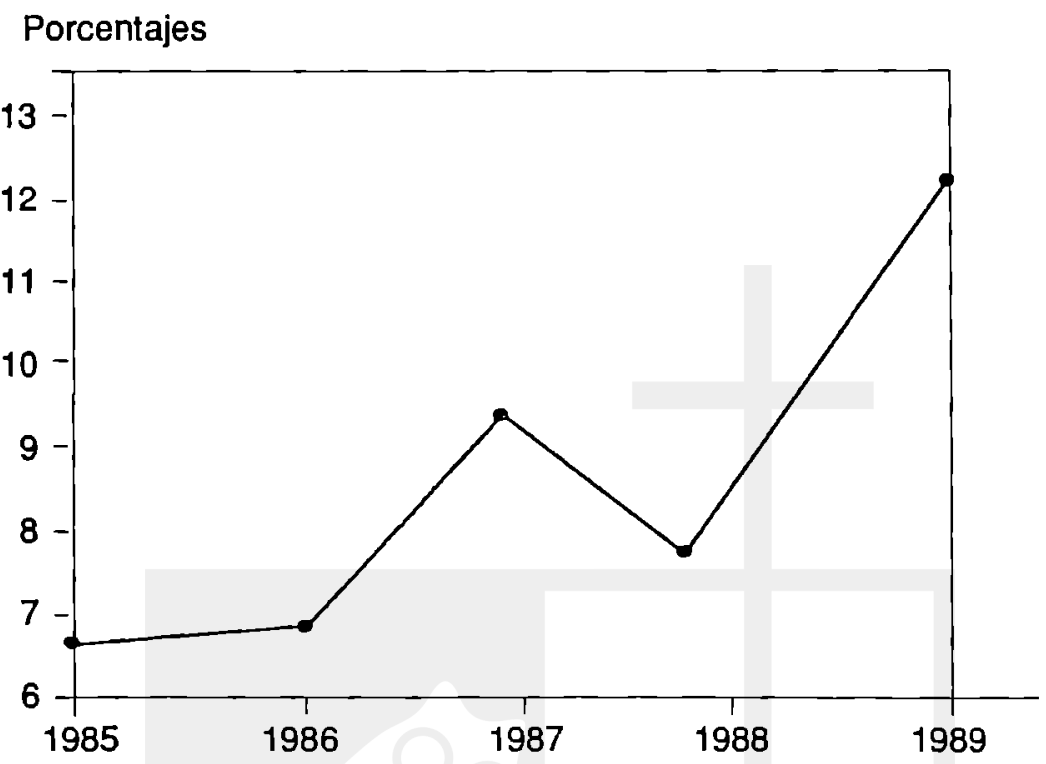

Fuenle: BCA

- Saldo en cuenta corriente (exlcuyendo donaciones)/PIB

bajo nivel en toda la década. Los esfuerzos realizado por el actual gobiemo a fin de estimular las exportaciones, como la privatización del comercio exterior del café y el azúcar, y las reformas al impuesto del café y eliminación del impuesto a las exportaciones del algodón y azúcar, no parecen haber rendido aún los frutos esperados. Algunas de estas medidas no han hecho sino contribuir a la reducción de los ingresos tributarios y al agravamiento del déficit fiscal.

El impacto de la liberalización de las importaciones, tanto en la forma de reducción de aranceles como de la eliminación de una serie de trabas y obstáculos administrativos que habian estado vigentes durante buena parte de la década, se ha reflejado ya en un incremento sustancial de las importaciones de bienes (cuadro 4) y en la consiguiente presión sobre el tipo de cambio. El desmesurado aumento que, como veremos más adelante, se produjo este año en el déficit fiscal ha contribuido también al deterioro de la cuenta corriente.

En cuanto a la cuenta de capilal, es interesante observar el com- 
portamiento del capital privado, cuya salida neta duplica el ingreso neto de capital bancario y anda muy cerca de las entradas por concepto de capital oficial (cuadro 4). Al parecer, ni las medidas de política cambiaria y financiera ni la confianza que el actual gobierno pueda inspirarles, resultan suficiente para convencer a los grandes empresarios salvadorefíos sobre la conveniencia de repatriar sus capitales, o al menos frenar sus salida del país. La continuación de la guerra es sin duda un factor que pesa más para ellos.

Es interesante observar también la fuerte cantidad de pagos diferidos que el gobierno ha llegado a acumular, de manera especial en 1989 (cuadro 4), algo que no parece estar desvinculado de las dificultades que éste estaría teniendo para honrar sus compromisos financieros internacionales, ante la crítica situación de disponibilidad de divisas.

\subsection{Tlpo de camblo}

La precaria situación de la balanza de pagos y la pérdida de reservas internacionales ha tenido como una de sus manifestaciones más visibles una evolución ascendente del tipo de cambio, sobre todo en el mercado extrabancario (gráfico 5). Como ya hemos sefialado, el tipo de cambio se habria devaluado en por to menos un $25 \%$ durante el anio. Esto ha obedecido tanto a la presión a que se ha visto sometida la limitada oferta de divisas, presión que se ha visto incrementada a raíz de la liberalización parcial de las importaciones, como a la puesta a flotación del dólar a partir del mes de julio.

Vale la pena formular algunas observaciones sobre el problema cambiario durante 1989. En primer lugar, cabe señalar los enormes estuerzos y sacrificios que las autoridades monetarias han tenido que realizar mediante una politica crediticia sumamente restrictiva hacia el sector privado, a fin de impedir un disparamento del precio del dólar, por temor sobre todo a los efectos inflacionarios que ésto provocaría, como también a la mayor especulación a que daria lugar. Esta política, como ya hemos sefialado, ha tenido costos nada desdefiables en terminos de crecimiento económico y desempleo. Ha sido, en todo caso, esta política restrictiva la que ha evitado en buena medida un alza desmesurada del tipo de cambio, y posibilitado una estabilidad relativa del mismo. La intervención de las autoridades monetarias en este sentido, ha configurado de hecho un régimen cambiario de flotación sucia.

En segundo lugar, pese a los esfuerzos realizados por las autoridades monetarias, la brecha entre el tipo de cambio bancario u oficial y el prevaleciente en el mercado negro mostró una tendencia a 


\section{Gráfico 6}

\section{COTIZACION EXTRABANCARIA DEL DOLAR 1989}

Colones por dólar

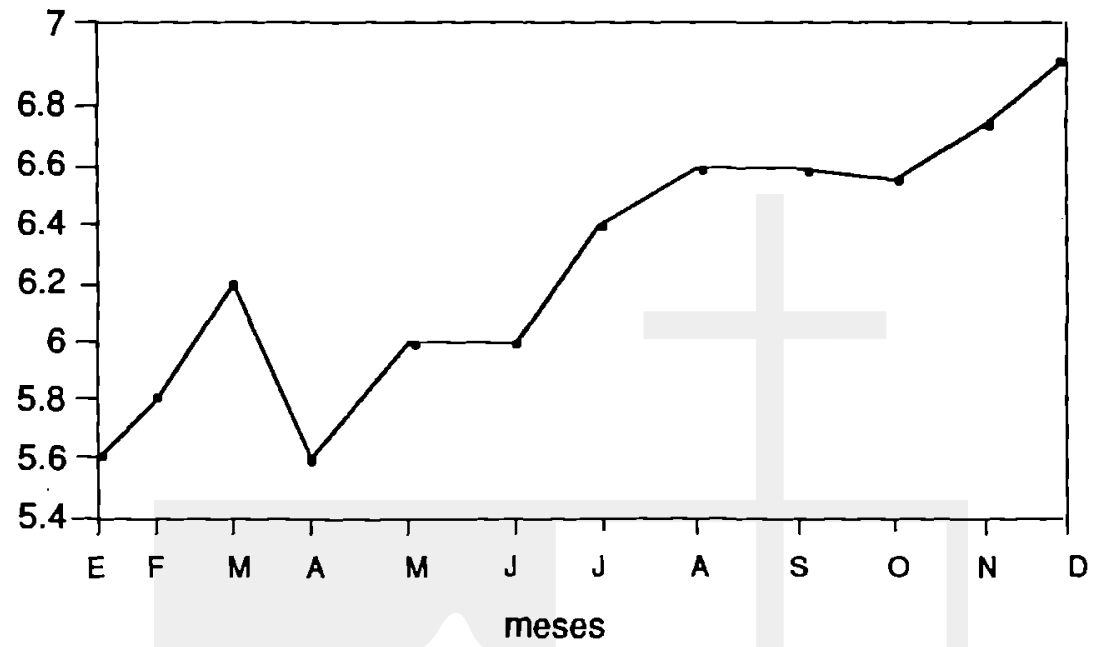

ampliarse en el último bimestre del año, al parecer de manera un poco independiente de los acontecimientos bélicos de noviembre en la capital. Esta tendencia al ensanchamiento ha continuado durante el primer mes de 1990, y pareciera estarse profundizando. Aunque el Banco Central no parece dispuesto a mantener el tipo de cambio a un nivel específico por períodos más o menos largos cuando en el mercado negro la tendencia es hacia el alza, la ampliación de esta brecha podría ir configurando una situación similar a la existente antes de julio pasado, en donde el precio del dólar en el mercado negro se mantuvo de manera permamente varias décimas por encima de su precio oficial. Este era, por cierto, uno de los inconvenientes que las nuevas autoridades veian en el antiguo régimen cambiario, y que supuestamente habría de desaparecer - junto con el mercado negro-, una vez implementada la flotación.

El ensachamiento de esta brecha no contribuye sino a una alza en el futuro inmediato del tipo de cambio oficial, no necesariamente por la existencia de la brecha en si, sino porque conduce a una reducción de la oferta de divisas en los bancos. Por otra parte, constituye un inconveniente bastante serio para las autoridades económicas que han optado preferencialmente por la promoción de exportaciones como núcleo del crecimientu económico, pues los exportadores están obli- 
gados a vender sus divisas a los bancos, pero no necesariamente autorizados a comprarlas libremente en ellos. De esta manera, no hacen sino subsidiar a cierto tipo de importadores.

Aunque, por hoy, las autoridades han sido capaces de mantener bajo cierto control el tipo de cambio -inclusive el del mercado negrono parece muy probable que ésto vaya a continuar por mucho tiempo. De hecho, el sustancial aumento que el dólar ha sufrido en las primeras semanas de 1990, sugiere que el gobierno va perdiendo su capacidad de control relativo sobre el tipo de cambio. La reducción de la ayuda norteamericana para 1990; la suspensión y/o retención de ese tipo de ayuda por parte de algunos países europeos ante el deterioro de la situación de los derechos humanos en el pais; la prolongación del desacuerdo sobre cuotas en la OIC por al menos un año más y la consiguiente continuación de bajos precios para el café, así como la mayor reducción de aranceles programada para 1990, hacen preveer no sólo un aumento sustancial del tipo de cambio, sino también la pérdida del control relativo que sobre el han ejercido hasta ahora las autoridades monetarias.

Aunque para 1989 la tasa de devaluación de la moneda fue sin duda superior a la de inflación, no parece que ésto haya tenido repercusiones visibles de importancia en términos de competitividad, que obviamente, en el caso de El Salvador, no es simplemente un problema de tipo de cambio sobrevaluado. El tipo de cambio real, medido por el tipo de cambio de paridad, habría aumentado en cerca de un $15 \%$; medido por la relación de precios bienes comerciados y no comerciados internacionalmente, habria caído en casi un $10 \%$. Estos datos aparentemente conflictivos estarian indicando to siguiente: si bien la competitividad del país habría aumentado a raíz de la devaluación, este aumento no habría sido lo suficientemente importante como para inducir en el mediano plazo a una reasignación de recursos del sector productor de bienes no comerciados hacia el de bienes comerciados internacionalmente, de modo que el sesgo antiexportador de la economía no habría ni siquiera disminuido.

\section{Sector público}

No menos grave que el deterioro de la situación de la balanza de pagos habria sido la agudización de la crisis fiscal. En tajante contraste con los planes y promesas de las actuales autoridades económicas, la situación fiscal del país se habría agravado de manera considerable en 1989. Según estimaciones del Ministerio de Hacienda, el déficit del gobierno central habria ascendido a más de 1107 millones de colones 


\section{Cuadro 5}

Goblerno central: operaciones consolidadas

(millones de colones)

\begin{tabular}{lrrrr}
\hline & 1986 & 1987 & 1988 & 19891 \\
\hline 1. Ingresos Corrientes & 2846.0 & 2753.4 & 2843.8 & 2616.4 \\
2. Gastos Corrientes & 2567.2 & 2761.8 & 3042.2 & 3480.5 \\
3. Ahorro Corriente & 278.8 & -8.4 & -198.4 & -864.1 \\
4. Donaciones & 314.2 & 545.7 & 542.0 & 432.0 \\
5. Gastos de Capital & 601.9 & 703.4 & 588.0 & 679.9 \\
6. Concesión Neta de Préstamos & 269.2 & 127.6 & 60.0 & -4.6 \\
7. Déficit Fiscal (5+6-3-4) & 278.1 & 293.7 & 304.4 & -1107.4 \\
B. Financiamiento Neto & 278.1 & 293.7 & 304.4 & 1107.4 \\
A. Interno & -43.0 & 14.2 & 26.4 & 982.8 \\
$\quad$ Banco Central-Crédito Neto & -301.5 & 84.4 & -69.5 & 888.1 \\
$\quad$ Banco Com.-Crédito Neto & 114.6 & 58.3 & -92.8 & 97.2 \\
$\quad$ Otras fuentes & 143.9 & -128.5 & 188.8 & -2.5 \\
B. Externo & 321.1 & 279.5 & 278.0 & 124.6 \\
\hline
\end{tabular}

Fuente: Ministerio de Hacienda.

1. Estimaciones.

Gráfico 7

\section{RELACION DEFICIT FISCAL/PIB (\%)}

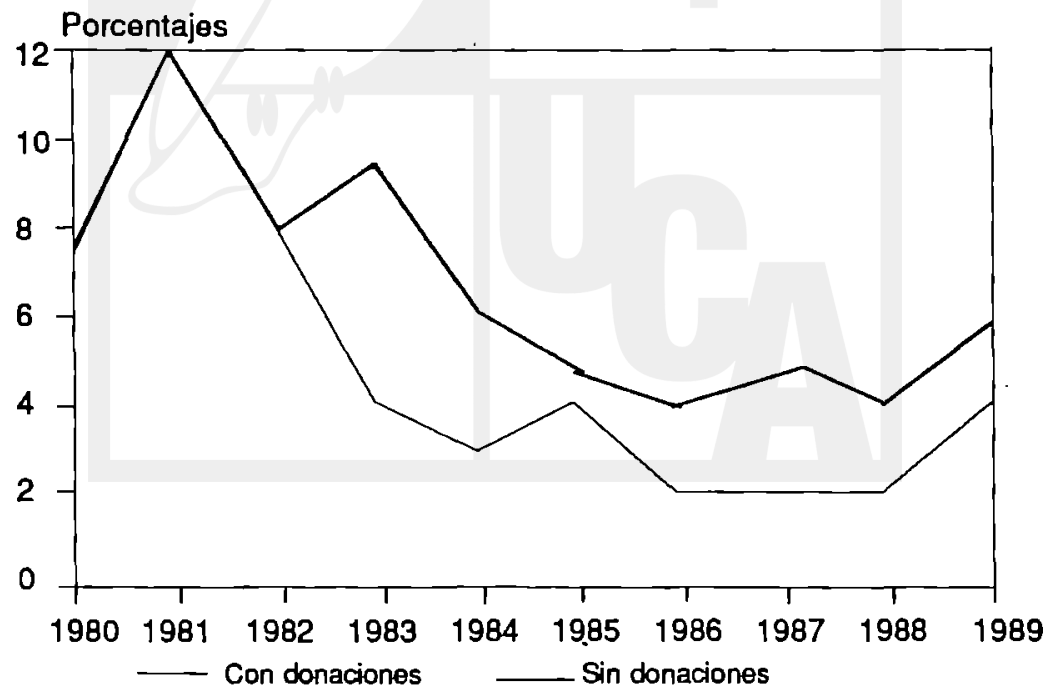

Fuente: Ministerio de Hacienda.. 
(3.6 veces superior al del año anterior), si se incluyen las donaciones recibidas del exterior, y a 1539 (2 veces mayor que en 1988) millones excluyendo donaciones (véase cuadro 5). Estas cifras son las más altas registradas en la historia económica del país. Este desmesurado aumento hizo que la relación déficit fiscal/PIB (exlcuyendo donaciones), ascendiera a casi 5\%, la más alta desde 1983 (gráfico 7).

Según fuentes oficiales, el agravamiento de la situación fiscal respondió al incremento salarial y menores ingresos originados por la caída de los precios del café en el mercado internacional, el no pago de los impuestos por parte del INCAFE y a una sobreestimación de los posibles ingresos de la lotería instantánea, así como a los gastos adicionales en defensa y seguridad a raíz de la ofensiva guerrillera de noviembre-diciembre. El incremento del servicio de la deuda interna y externa, así como los menores ingresos asociados a la reforma a el impuesto a la exportación de café, influyeron también sobre los resultados de 1989.

Aunque la situación fiscal sufrió sin duda un sensible deterioro, visto únicamente desde la perspectiva de la relación déficit fiscal/PIB, el problema no parece ser de por sl tan grave. Esta relación, que desde 1983 había descendido sustancialmente (gráfico 7) y ha caido a niveles inferiores a los de 1976-1978, se encuentra dentro de un margen juzgado tolerable por organismos como el FMI. El problema, sin embargo, es la manera en que el déficit es financiado. Contrastando fuertemente con la tendencia observada desde 1986 (cuadro 5), el financiamiento del déficit por el BCR alcanzó la alarmante cifra de 888.1 millones de colones, una nueva marca en la historia económica del pais y un verdadero modelo de irresponsabilidad fiscal, que muy pocos podrían haber esperado del actual gobierno. (Es durante la segunda mitad del af́o que el financiamiento del BCR cobra dimensiones alarmantes). Resulta un poco sorprendente que la inflación haya alcanzado un nivel bastante moderado y que inclusive haya disminuido respecto al afio anterior con una creación de dinero de tal magnitud ${ }^{8}$.

El crédito neto otorgado por los bancos comerciales aumentó también de manera significativa (cuadro 5), lo que en un marco de recursos financieros limitados no implica otra cosa sino desplazamiento de la inversión privada (como ya vimos el crédito al sector privado cayó en más de un $14 \%$ en términos reales), algo que tan asiduamente han criticado en los últimos años quienes ahora conducen la politica económica.

Es interesante observar la notable reducción en recursos externos (distintos de donaciones), ocurrida en 1989, disminución que, aunque 
sigue una tendencia de 2 años atrás, está sin duda vinculada a las dificultades que el actual gobierno enfrenta para la consecución de ayuda externa, derivadas en buena medida de su pobre imagen internacional.

El gobiemo se muestra empefiado en reducir el déficit fiscal, pero casi exclusivamente vía recorte de gastos. Sin embargo, el espacio de maniobra con que cuenta al respecto es bastante reducido, a menos que esté dispuesto a asumir los serios costos políticos que ello acarrearía. Para 1989, más del $80 \%$ de su gasto total estuvo destinado a gastos de funcionamiento (remuneraciones + compra de bienes y servicios) y a honrar el servicio de su deuda, erogaciones que son obviamente bastante rigidas y difíciles de reducir en el corto plazo. Las remuneraciones evidentemente pueden reducirse sólo a costas de aumentar el desempleo y agudizar el descontento social, algo que -sobre todo ante la celebración de elecciones el año próximo- probablemente no todos en el gobierno estén anuentes a aceptar. No obstante, en los últimos meses de 1989 y primeras semanas de 1990 han tenido lugar algunos despidos masivos, gracias en parte a que el gobierno ha podido silenciar las protestas de las gremiales sindicales a causa de la vigencia del Estado de Sitio. El servicio de la deuda, por otra parte, es algo que se encuentra bastante fuera de control del gobierno.

Ante la rigidez de estos rubros, los componentes del gasto cuya reducción sería, al menos teóricamente, más factible para el gobierno son el de transferencias y el de formación de capital. En parte, dadas las conseucencias económico sociales, sobre todo a mediano plazo, que el recorte de la formación de capital acarreraría, el gobierno parece haber optado por recortar las transferencias, en particular aquellas destinadas al resto del sector público. Se trata, concretamente, de las transferencias al resto del gobierno general y a las empresas públicas no financieras, que en 1988 representaron el $12.6 \%$ del gasto y en 1989 el $\mathbf{8 . 4 \%}$. En este contexto se inscribiría el incremento de las tarifas públicas, iniciado en la segunda mitad del año. Las transterencias corrientes al sector privado también habrian de reducirse mediante el recorte o eliminación de subsidios.

Muchísimo menos interés se ha puesto en la necesidad de aumentar los ingresos tributarios. De hecho, los planes del gobierno son ir reduciendo paulatinamente la carga tributaria, que ya en 1989 habria alcanzado su más bajo nivel en toda la década. Esto, en un contexto de estancamiento económico como el vivido en los últimos años, no implica sino una reducción de los ingresos tributarios. Obviamente, el gobierno pretende darse este lujo a costa de recortar el gasto. Las reformas 
Gráfico 8

EVOLUCION DE LOS INGRESOS CORRIENTES

$Y$ GASTOS TOTALES REALES $(1985=100)$

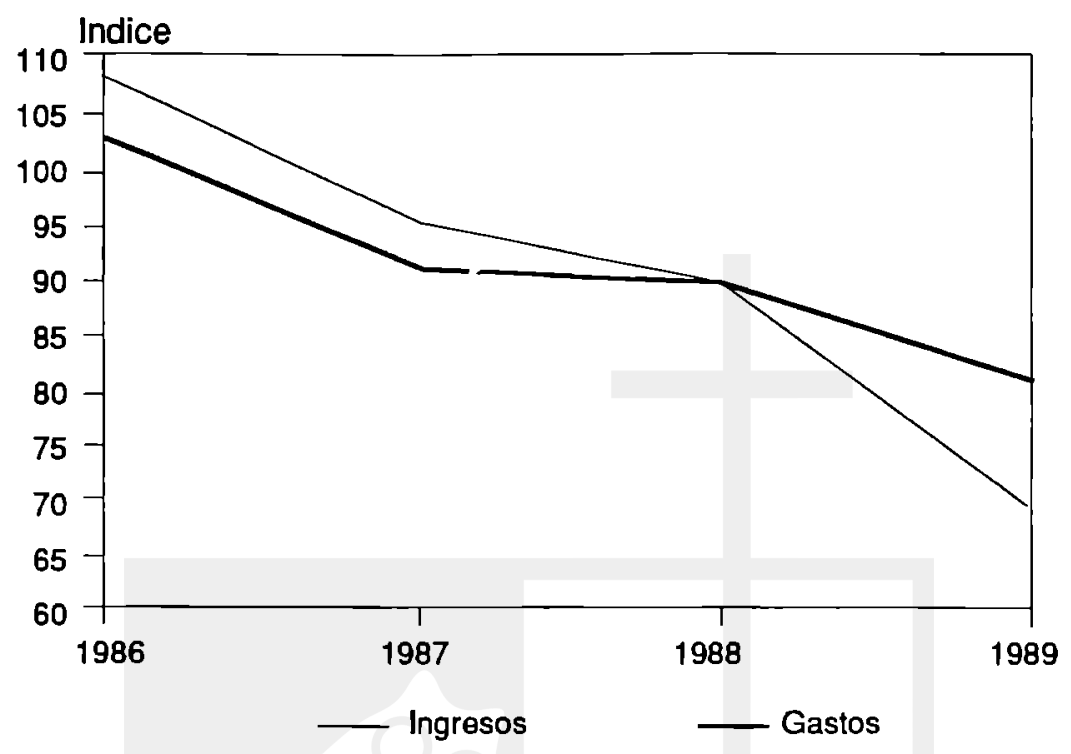

Fuente: Ministerio de Hacienda.

tributarias que ya han comenzado a implementarse no tienen como prioridad aumentar los ingresos tributarios, sino más bien reducir la carga impositiva - sobre todo para los sectores de más altos ingresosa fin de estimular la inversión y/o las exportaciones. Estas reformas van a agudizar sustancialmente el carácter regresivo e inequitativo del sistema tributario salvadorefio, sin que ello vaya a significar necesariamente un aumento en los ingresos tributarios o frenar al menos su caida en términos reales. No es otra cosa lo que el presupuesto de 1990 refleja ${ }^{7}$.

El problema radica en que el gobierno concibe la crisis fiscal como un problema de gastos meramente, mientras que es en lo fundamental un problema de ingresos. Ya hemos visto (gráfico 7) como el gasto como proporción del PIB ha caído notablemente desde 1983, y, por tanto, como la participación del Estado en la economía, contrario a lo que las actuales autoridades económicas sostienen, se ha venido reduciendo. En el gráfico 8 podemos ver también como el gasto público real a caído persistentemente desde 1986. Lo mismo ha sucedido con los ingresos corrientes reales; solo que estos últimos han venido cayendo a un ritmo bastante mayor (gráfico 8 ). 
El gobierno no sólo ha ignorado esta evidencia, sino también las recomendaciones que estudios cuidadosos y serios han formulado al respecto. En un estudio reciente - realizado por cierto con el patrocinio de FUSADES - sobre el tamaño e incidencia del sector público salvadorefio se ha planteado el problema de manera sumamente clara: "...a nivel macroeconómico, la crisis de la tinanzas públicas del país y el déficit fiscal no se debe a un excesivo gasto fiscal... la falla del gobierno ha sido recaudar suficientes ingresos tributarios como para financiar el gasto"'.

\section{Conclusión}

Pese a que la economía salvadoreña experimentó en 1989 un leve crecimiento global, la crisis económica en que el pais se encuentra sumergido desde hace diez años no sólo no pudo ser frenada, sino que se agravó. El ingreso por habitante habría descendido nuevamente, mientras que el proceso inflacionario no da muestras claras de que haya empezado a ser efectivamente controlado. Como resultado, el deterioro de los niveles de vida para las grandes mayorias siguió su curso acelerado. La crisis en las finanzas públicas y en el sector externo, por otra parte, se habria agravado sensiblemente.

Obviamente, estos magros resultados no obedecen sólo o necesariamente a un mal manejo de la política económica. Las características estructurales de la economía salvadoref́a y la continuación del conflicto bélico, factores que no sólo reducen enormemente el margen de maniobra de cualquier gestión económica, sino que imponen límites infranqueables a cualquier proceso de recuperación y estabailización de la economia, parecen ser los principales factores en la explicación del pobre desempeño económico de El Salvador en 1989.

\section{NOTAS}

1. Cfr. Roberto Rivera (1989). "Producción de alimentos y desbalances macroeconómicos". Realldad Económico-Soclal 2, N 1 (septiembre-octubre), pp. 437-55.

2. MIPLAN (1989). Programe de desarrollo económlco y social 1989-1994, pp. 26 y 93.

3. No contamos con dalos sobre la distribución exacta del manejo de divisas entre el mercado oficial y el mercado negro. Teniendo en cuenta, sin embargo, que el tipo de cambio en el mercado oficial subió de 5 a 6.45 entre enero y diciembre (un incremento de $29 \%$ ), mientras que en el mercado negro de 5.6 a 6.8 (un alza de $21.4 \%$ ), una devaluación general de aproximadamente un $25 \%$ no serla una citra muy alejada de le realidad, aunque podrla ser un poco conservadora. 
4. Cfr. Roberto Rivera (1988). "La inflación en El Salvador". Realldad Económico-Soclal Ne 1 (enero-febrero), pp. 1-42.

5. Este punto ha sido claramente planteado en S. Fischer (1986). "Issues in MediumTerm Macreconomics Adjustment". World Observer 1, pp. 163-82.

6. Datos como éste hacen durar a muchos sobre la veracidad de la cifra de inflación dada a conocer oficialmente.

7. Véase Proceso 406, pp. 10-12.

8. D. Wisecarver (1989). El temafio e Infiuencla del sector públlco en la economla salvadoref́a. San Salvador: FUSADES, p. 32.

Digitalizado por Biblioteca "P. Florentino Idoate, S.J." Universidad Centroamericana José Simeón Cañas 